\title{
Modulation of SARS-CoV-2 Spike-induced Unfolded Protein Response (UPR) in HEK293T cells by selected small chemical molecules
}

\author{
Balakrishnan $\mathrm{B}^{*}$, Lai $\mathrm{K}^{*}$
}

Division of Medical Genetics, Department of Pediatrics, University of Utah

* Corresponding Author 


\begin{abstract}
Coronaviruses (CoV) exploits the endoplasmic reticulum (ER) of the host cells for replication and in doing so, increases ER stress. evokes Unfolded Protein Response (UPR) and possibly autophagy, which could all attribute to the pathophysiology of the viral infections. To date, little is known about the roles of ER stress, UPR, and autophagy in SARS-CoV-2 infection. Here we over-expressed the viral Spike (S) protein in cultured HEK293T cells, as it has been shown that such protein is largely responsible for UPR activation in other CoV-infected cells. We noticed, in the transfected cells, heightened ER stress, activation of the PERK-elF2 $\alpha$ arm of the UPR, induction of autophagy and cell death. When we treated the transfected cells with Tauroursodeoxycholic acid (TUDCA), 4-phenyl butyric acid (PBA), Salubrinal, Trazadone hydrochloride, and Dibenzoylmethane (DBM), we saw reduced the BiP/GRP78 levels, but only PBA and TUDCA could significantly diminish the levels of pelF $2 \alpha$ and autophagy expression.
\end{abstract}


bioRxiv preprint doi: https://doi.org/10.1101/2021.02.04.429769; this version posted February 4, 2021. The copyright holder for this preprint (which was not certified by peer review) is the author/funder. All rights reserved. No reuse allowed without permission.

\section{Keywords}

SARS-CoV-2

COVID-19

\section{Endoplasmic reticulum stress}

Unfolded protein response

PERK/elF2 $\alpha$

Autophagy 


\section{Introduction}

Coronavirus disease 2019 (COVID-19) is a potentially lethal, debilitating infectious disease caused by the newly discovered coronavirus called SARS-CoV-2 [1]. Like other coronaviruses, SARS-CoV-2 is an enveloped and positive-sense single-stranded RNA virus with a large genome of $\sim 30 \mathrm{~kb}$. Morphologically, coronaviruses are spherical or pleomorphic in shape with a mean diameter of 80-120 nm [2]. They are characterized by the large $(20 \mathrm{~nm})$ "club-like" projections on the surface, which are the heavily glycosylated trimeric Spike (S) proteins [2, 3]. Coronavirus infection is initiated by the binding of the $S$ protein to the angiotensin-converting enzyme 2 (ACE2) cell surface receptors followed by fusion of the virus and cell membrane to release the viral genome into the cell $[4,5]$. The S protein comprises two functional subunits, S1 (bulb) for receptor binding and S2 (stalk) for membrane fusion. The interaction between the host ACE2 cell surface receptor and the S1 subunit is the central element of coronaviruses' tropism [6]. It replicates in the cytoplasm, and its life cycle is closely associated with the endoplasmic reticulum (ER). The viral activities thus have a profound impact on ER functions. Particularly, SARS-CoV-2 hijacks the ER to process its structural and nonstructural proteins.

The accumulation of nascent and unfolded viral secretory and structural proteins in the ER lumen can lead to ER stress and the downstream activation of multiple signaling pathways [7]. To accommodate the biosynthetic encumbrance and capacity of the ER for maintaining cellular homeostasis, the host cell activates Unfolded Protein Response (UPR) to mitigate the ER stress. The UPR comprises three critical signaling pathways initiated by localized stress sensors in ER, such as inositol-requiring enzyme 1 (IRE-1), 
activating transcription factor 6 (ATF6), and PKR-like ER kinase (PERK) [8]. These proteins send robust signals to the cytosol and nucleus to alleviate the burden of misfolded protein and ensure successful ER protein homeostasis [9]. However, undue or prolonged activation of UPR can also trigger autophagy and apoptosis [10], which could play a significant role in the pathophysiology of viral infections. For SARS-CoV (the coronavirus that caused SARS), it was reported that the Spike (S) protein, but not the envelope $(E)$, membrane $(M)$, or the nucleocapsid $(N)$ protein evokes UPR by the transcriptional activation of GRP78 (BiP)/GRP94 and upregulation of the PERK pathway with no effects on the other two arms of UPR [11-13]. Studies also confirmed UPRtriggered autophagy of the host cells in SARS-COV and MERS-COV infections [14, 15]. Based on the previous studies on other coronaviruses, it is reasonable to assume that SARS-CoV-2 may induce autophagy via UPR activation in the infected cells. However, emerging findings suggest that SARS-CoV and COVID-19 are also unique in many ways. For instance, short-term loss of smell and taste during the infection cycle [16-19], abnormal coagulation [20-22], silent hypoxia [23], and rash [24-26] in some patients with the new COVID-19 are just some of the unique phenotypes that were rarely reported for other coronaviral illnesses. Such unique phenotypes are telltale signs that it is premature to assume everything we learned from other coronaviruses, including SARS-CoV, can be applied in wholesale to SARS-CoV-2 without further investigations.

In this short communication, we aim to study the impact of the over-expression of the Spike (S) protein of SARS-CoV-2 in HEK293T cells on ER stress manifestation and its downstream effects, including autophagy. Puelles and coworkers recently reported the SARS-CoV-2 tropism in human kidney by detecting the presence of ACE2 mRNA in 
the kidney cells, as well as the enhanced expression of transmembrane serine protease 2 (TMPRSS2) and cathepsin L (CTSL), which are also considered to facilitate SARSCoV-2 infection in multiple kidney-cell types from fetal development through adulthood [27]. Indeed, acute kidney injury and dysfunctions are observed in a large proportion of COVID-19 patients [28-31]. Hence the use of HEK293T cells in this study is of significant clinical relevance. Our results showed that over-expression of the viral Spike protein in the HEK293T cells activated UPR, which led to autophagy. Interestingly, the activated UPR and the downstream autophagy induced could be substantially diminished by selected UPR modulators, some approved by the U.S. Food and Drug Administration (FDA) for treating other diseases [32-35]. 


\section{Materials and Methods}

\subsection{Cells, Antibodies, and Reagents}

HEK293T cells obtained from ATCC (Manassas, VA) were cultured in a $37^{\circ} \mathrm{C}, 5 \%$ $\mathrm{CO}_{2}$ incubator in Dulbecco's Modified Eagle Medium (DMEM) supplemented with $10 \%$ (vol/vol) FBS, $10 \mathrm{mM}$ HEPES, $100 \mathrm{lU} / \mathrm{mL}$ penicillin, and $100 \mu \mathrm{g} / \mathrm{mL}$ streptomycin.

Anti-GRP78 (BiP), anti-pelF2 $\alpha$, anti-LC3B, and anti-GAPDH were purchased from Cell Signaling Technology Inc. (Danvers, MA). Goat anti-rabbit IRDYE 800CW was purchased from Li-COR Biosciences. (Lincoln, NE). anti-SARS-CoV-2 Spike Protein was purchased from Origene Technologies Inc. (Rockville, MD).

Transfection reagent Lipofectamine ${ }^{\circledR} 3000$ transfection reagent was purchased from Thermo Fisher Scientific Inc. (Waltham, MA).

Cell Proliferation Kit I (MTT) was obtained from Sigma- Aldrich Inc. (St. Louis, MO).

Tauroursodeoxycholic acid (TUDCA), 4-phenyl butyric acid (PBA), Salubrinal, Trazadone hydrochloride, and Dibenzoylmethane (DBM) were purchased from SigmaAldrich Inc. (St. Louis, MO).

\subsection{Plasmid}

pcDNA3.1-SARS2-Spike, which was constructed by Fang Li (Addgene plasmid \# 145032; http://n2t.net/addgene:145032; RRID: Addgene_145032), was purchased from Addgene Inc. (Watertown, MA).

\subsection{Transfection Studies}


HEK293T cells were plated onto six-well plates at a density of $4 \times 10^{5}$ cells per well and cultured at $37^{\circ} \mathrm{C}$ with $5 \% \mathrm{CO}_{2}$ overnight for transfection. A total amount of $5 \mu \mathrm{gNA}$ per well was used for transient transfection with Lipofectamine $₫ 3000$ according to the manufacturer's protocol. Twenty-four hours post-transfection, culture media was changed, and the cells were extracted and lysed using MPER lysis buffer (Thermo Fisher Scientific Inc.). For the drug treatment, the UPR modulators at the indicated concentrations were administered 4 hours post-transfection, and proteins from the cells were extracted after 24 hours of treatment.

\subsection{Western Blot Analysis}

Cells overexpressing C9-tagged Spike protein and the drug treatment group were harvested 24- and 28-hours post-transfection respectively. The cells were lysed with 0.1 $\mathrm{ml}$ of MPER buffer containing $1 \mathrm{mM} \mathrm{NaVO3,} 2.5 \mathrm{mM}$ sodium pyrophosphate, $1 \mathrm{mM} \beta$ glycerol phosphate, $1 \mathrm{mM}$ phenylmethylsulfonyl fluoride, $5 \mu \mathrm{g} / \mathrm{ml}$ aprotinin, and $5 \mu \mathrm{g} / \mathrm{ml}$ leupeptin, $(\mathrm{pH} 7.5) .30 \mu \mathrm{g}$ of total protein from cell lysate was separated on $12 \%$ sodium dodecyl sulfate-polyacrylamide gel electrophoresis, electro-transferred to nitrocellulose membrane (LI-COR). Antibodies described above were used to probe the membranes according to the protocols the manufacturer provided. The signals were detected by Infrared detection using an Odyssey scanner and analyzed by Image Studio software (LICOR).

\subsection{Detection of Autophagy}


For the microtubule-associated protein 1A/1B-light chain 3 (LC3) mobility shift assay, SARS-CoV-2 Spike protein-expressing cells were washed with cold PBS, lysed with MPER buffer, and subjected to Western blot analysis with antibodies against LC3B. LC3-I is about $16 \mathrm{kD}$, and lipidated LC3 (LC3-II) is about $14 \mathrm{kD}$. The level of LC3 II formation represents the autophagic activity [36].

\subsection{Cell viability determination by MTT assay}

Cell viability was assessed by an MTT assay. Briefly, HEK293T cells were seeded in 96 -well plates at a density of $5 \times 10^{4} /$ well. The cells were transfected with the plasmid, pcDNA3.1-SARS2-Spike as described above. 24 hours post-transfection, MTT solution (10 $\mu \mathrm{l}, 5 \mathrm{mg} / \mathrm{ml}$ in PBS) was added to each well and incubated at $37^{\circ} \mathrm{C}$ for 4 hours. Then, the medium was replaced by $100 \mu \mathrm{LMSO}$ per well. The plate was gently shaken for 5 minutes to completely dissolve the precipitate and incubated for 30 minutes. The absorbance was measured at $560 \mathrm{~nm}$ using a microplate reader. Cell viability was expressed as a percentage of the control.

\subsection{Quantification and Statistical Analysis}

All data were expressed as Mean \pm SEM as indicated. Statistical significance between the two groups was tested by the Student's t-test. P values of less than 0.05 were considered significant. 


\section{Results}

\subsection{UPR is activated upon SARS-CoV-2 Spike protein overexpression in cultured cells}

To study the molecular events subsequent to SARS-CoV-2 Spike overexpression in details, the plasmid expressing SARS-CoV-2 Spike protein was transiently transfected into cultured HEK293T cells at different DNA concentrations. Manifestation of ER stress was first demonstrated by evaluating the intracellular GRP78 levels in the transfected cells. In cells over-expressing the SARS-CoV-2 Spike protein, the level of BiP/GRP78 was significantly higher compared to the untransfected control (Fig. 1a). We next investigated which specific UPR pathways are activated upon overexpression of the Spike protein, with particular emphasis on the PERK arm. We found a significant increase in the levels of phosphorylated elF2a in the transfected cells 24 hours post-transfection (Fig. 1b). This further substantiated the hypothesis that SARS-CoV-2- Spike protein overexpression induces UPR in cultured cells.

\subsection{SARS-CoV-2 Spike protein expression triggers autophagy response in cultured cells}

There is growing evidence to show that various host cellular responses, including autophagy, innate immunity, and apoptosis, are affected or activated by coronaviruses infection in vivo [37-39]. Among the many signaling pathways, the UPR and autophagy are tightly interconnected and were shown to be essential for viral infection by multiple previous studies. The implication of autophagy in coronavirus infection has attracted 
substantial attention, due to the SARS-CoV outbreak in 2002-2003 [40-43]. To investigate the involvement of autophagy in cells over-expressing the SARS-CoV-2 Spike protein, we evaluated the molecular markers of autophagy by monitoring levels of LC3-I and LC3-II. We found that levels of LC3-II were increased in Spike protein-overexpressed cells from 24 hours post-transfection (Fig. 2a), which is indicative for an activated autophagy response that culminates in degradation cytoplasmic components in the lysosomes. Collectively, these data illustrated that the PERK/elF2a pathway of UPR is activated in SARS-CoV-2 infection, along with the induction of autophagy. To assess the cell viability by Spike protein overexpression, we conducted MTT assay 24 hours post transfection in HEK293T cells. The results revealed that SARS-CoV-2 Spike protein expression in HEK293T cells leads to cell death (Fig. 2b), which could be the consequence of UPR-induced autophagy shown above.

\subsection{UPR modulators reduced the UPR and autophagy in cultured cells}

We next selected a few UPR modulators, some of them were approved by the FDA to treat other diseases [32-35], in an attempt to modulate the level or ER stress and/or the UPR in HEK293T cells over-expressing the viral Spike protein. HEK293T cells were treated with 4-PBA, TUDCA, DBM, Trazadone, and Salubrinal at indicated concentrations 4 hours after plasmid encoding Spike protein transfection. The expressions of GRP78, pelF2 $\alpha$, and LC3-II were examined at 24 hours post-transfection. Salubrinal, TUDCA, PBA, and Trazadone significantly reduced the BIP/GRP78 levels in cells overexpressed with viral Spike protein (Fig. 3a). In addition, we saw a significant reduction in pelF2 $\alpha$ in cells treated with the PBA and TUDCA (Fig. 3b). Interestingly, treatment with PBA and 
TUDCA also resulted in a diminished level of the autophagy marker, LC3-II (14 kD) in the cells over-expressing Spike at 24 hours (Fig. 3c). Altogether, we demonstrated the cause-and-effect relationship between Spike protein-induced UPR and the subsequent autophagy. We further analyzed the cell survival with UPR modulators and the data confirmed the protective effects of TUDCA and PBA on cell survival (data not shown). 


\section{Discussion}

Since February 2020, our world has been embroiled in a pandemic that is unprecedent in scale. As of Dec $27^{\text {th }}, 2020$, there are more than 81 million people worldwide infected with SARS-CoV-2_and over 1.77 million deaths. This horrific loss of human lives and the emotional toll inflicted on the loved ones of the deceased will continue to rise until the spread of the disease is halted. At the same time, many survivors who were fortunate to escape death suffer from a host of complications such lung fibrosis or systemic organ damages that will take years to fully heal. In addition to the significant mortality and morbidity caused by COVID-19, millions of jobs have been lost in the U.S. alone since March. Therefore, there is an urgent need for the development of life-saving therapies and preventive measures for COVID-19. But to accomplish these goals, we must improve our understanding of the pathophysiological mechanisms of the disease.

Being a coronavirus, SARS-CoV-2 shares some similarities with other Coronaviruses, including SARS-CoV. While our current knowledge on existing human coronaviral diseases could offer important insights into SARS-CoV, emerging findings suggest that SARS-CoV-2 is also unique in many ways. Some of the phenotypes such as short-term loss of smell and taste [16-19], abnormal coagulation [20-22] seen in many patients with the new COVID-19 were rarely reported for other coronaviral illnesses. Therefore, it is premature to assume the knowledge we gained from the studies of other coronaviruses, including SARS-CoV, can be applied in entirety to SARS-CoV-2 without additional research.

In this study we focused on the potential roles of ER stress, UPR and autophagy in SARS-CoV-2 infection because such fundamental cellular processes have been shown 
to contribute to the pathophysiology of other diseases [44-47]. Also, in case of viral infections, the role of ER during viral replication is well-documented, and the activation of UPR has been reported in other coronaviruses-infected cells [48-51]. Therefore, the induction of ER stress and UPR activation is a crucial factor in virus-host interaction and can significantly influence the patient's antiviral response and [52-55].

To study the potential roles of UPR and autophagy in SARS-CoV-2 infections, we chose to overexpress the SARS-CoV-2 Spike (S) protein in cultured HEK293T cells because it has been shown in SAR-CoV that the $S$ protein is the viral protein largely responsible for activation of UPR [11-13]. Moreover, our focus on the HEK293T cells is clinically relevant due to the high evidence of acute kidney injury in patients with COVID19. The mechanism(s) of renal injury caused by SARS-CoV-2 has not yet been fully elucidated, although ER stress/UPR has been implicated in renal injury induced by ischemia-reperfusion and nephrotoxicity [56].

Here we showed that the SARS-CoV-2 Spike protein expression in the HEK293T cells led to up-regulation of BiP/GRP78 (Fig. 1) and the activation of the PERK branch of the UPR pathway. These findings corroborated with the positive detection of ER stress markers in the lung samples infected from COVID-19 patients with severe pneumonia [57]. Our data also agreed with the recent study that shows the SARS-CoV and MHVCoV infections activated PERK arm of UPR with subsequently increased phosphorylation of elF2 $\alpha$ in the host cells [58-60]. It should be noted that the PERK pathway regulates innate immunity by suppressing type 1 interferon (IFN) signaling [60]. Hence, it suggests that UPR does play a role in attenuating IFN responses and innate immunity in coronavirus-infected cells. 
Several lines of evidence demonstrated that ER stress and the associated UPR contributes to autophagy $[61,62]$. However, the specific UPR pathway activated to mediate autophagic activities, and whether cytoprotective autophagy or autophagic cell death is induced upon ER stress, is not entirely clear and probably varies with the different triggers of the UPR $[63,64]$. Nonetheless, an essential step in UPR-induced autophagosome formation is the phosphorylation of PERK/elF2 $\alpha$ [65]. Once phosphorylated, elF2 $\alpha$ can induce the production of LC3-II from LC3-I to induce autophagy [66]. To further investigate the potential roles of UPR and autophagy in SARSCoV-2, we examined the HEK293T cells over-expressing the Spike protein and found that the LC3-II protein level was significantly upregulated with Spike protein expression, leading to cell death (Fig. 2). Furthermore, the changes in LC3-II observed were significantly reversed by pretreatment with PBA and TUDCA (Fig. 3), indicating these compounds could block the autophagy activation induced by over-expression of the Spike protein. It is unclear why PBA and TUDCA are more effective than the other UPR modulators we selected in this study. However, PBA and TUDCA are chemical chaperones [32, 67-71] and they could act to minimize UPR induction earlier on rather than mitigating the downstream signaling pathway like Salubrinal and the others.

At present, clinical management of COVID-19 includes infection prevention (maskwearing, social distancing, vaccination)supportive care (supplemental oxygen and mechanical ventilatory support) and limited and selective use of new anti-viral therapeutics [Remdesivir (Veklury) [72], convalescent plasma therapy with mixed results [73]. Dexamethasone, a glucocorticoid used to reduce inflammations in patient cells, has shown some promises in reducing the risk of death for patients with more severe SARS- 
CoV-2 infections, although the molecular mechanisms involved remain unclear. However, it has been shown that such glucocorticoid ameliorates ER stress in intestinal secretory cells with protein misfolding in vitro and in vivo [74]. Therefore, our current studies support the hypothesis that Dexamethasone might act through the modulation of UPR in the infected cells of the COVID-19 patients. Given the potential side-effects of prolonged use of glucocorticoids [75], one might want to consider the alternate use of other UPR modulators such as PBA and TUDCA, in combination of other approved antivirals. 


\section{Conclusions}

We show for the first time that over-expression of the Spike (S) protein of SARSCoV-2 heightened ER Stress, activated UPR and caused autophagy in human HEK293T cells in culture. Such cellular responses were significantly diminished by specific chemical chaperones PBA and TUDCA, but not other UPR modulators. These findings render significant implications in the pathophysiology and the treatment of the viral infection. 


\section{References}

1. Huang, C., et al., Clinical features of patients infected with 2019 novel coronavirus in Wuhan, China. Lancet, 2020. 395(10223): p. 497-506.

2. Wu, F., et al., A new coronavirus associated with human respiratory disease in China. Nature, 2020. 579(7798): p. 265-269.

3. Masters, P.S., The Molecular Biology of Coronaviruses. Adv Virus Res. , 2006. 66: p. 193292.

4. Alexandra C. Walls, et al., Structure, Function, and Antigenicity of the SARSCoV-2 Spike Glycoprotein. Cell, 2020. 180: p. 281-292.

5. Peng Zhou, X.-L.Y., [...]Zheng-Li Shi, A pneumonia outbreak associated with a new coronavirus of probable bat origin. Nature, 2020. 579: p. 270-27.

6. Hoffmann, M., et al., SARS-CoV-2 Cell Entry Depends on ACE2 and TMPRSS2 and Is Blocked by a Clinically Proven Protease Inhibitor. Cell, 2020. 181(2): p. 271-280 e8.

7. He, B., Viruses, endoplasmic reticulum stress, and interferon responses. Cell Death Differ, 2006.

13(3): p. 393-403.

8. Cox, J.S. and P. Walter, A novel mechanism for regulating activity of a transcription factor that controls the unfolded protein response. Cell, 1996.

87(3): p. 391-404.

9. Hetz, C.A. and C. Soto, Stressing out the ER: a role of the unfolded protein response in prion-related disorders. Curr Mol Med, 2006. 6(1): p. 37-43.

10. Sano, R. and J.C. Reed, ER stress-induced cell death mechanisms. Biochim Biophys Acta, 2014. 1833(12).

11. Chan, C.-P., et al., Modulation of the unfolded protein response by the severe acute respiratory syndrome coronavirus spike protein. J Virol

2006. 80(18): p. 9279-87.

12. To Sing Fung, M. Huang, and D.X. Liu, Coronavirus-induced ER stress response and its involvement in regulation of coronavirus-host interactions. Virus Res, 2014. 194: p. 110123.

13. Kam-Leung Siu, et al., Comparative analysis of the activation of unfolded protein response by spike proteins of severe acute respiratory syndrome coronavirus and human coronavirus HKU1. Cell \& Bioscience volume 2014. 4(3): p. 2045-3701.

14. Liao, Y., et al., Upregulation of CHOP/GADD153 during coronavirus infectious bronchitis virus infection modulates apoptosis by restricting activation of the extracellular signalregulated kinase pathway. J Virol, 2013. 87(14): p. 8124-34.

15. Versteeg, G.A., et al., The coronavirus spike protein induces endoplasmic reticulum stress and upregulation of intracellular chemokine mRNA concentrations. J Virol, 2007. 81(20): p. 10981-90.

16. Abalo-Lojo, J.M., J.M. Pouso-Diz, and F. Gonzalez, Taste and Smell Dysfunction in COVID19 Patients. Ann Otol Rhinol Laryngol, 2020: p. 3489420932617.

17. Dell'Era, V., et al., Smell and taste disorders during COVID-19 outbreak: A cross-sectional study on 355 patients. Head Neck, 2020. 
18. Gautier, J.F. and Y. Ravussin, A New Symptom of COVID-19: Loss of Taste and Smell. Obesity (Silver Spring), 2020. 28(5): p. 848.

19. Xydakis, M.S., et al., Smell and taste dysfunction in patients with COVID-19. Lancet Infect Dis, 2020.

20. Xiong, M., X. Liang, and Y.D. Wei, Changes in blood coagulation in patients with severe coronavirus disease 2019 (COVID-19): a meta-analysis. Br J Haematol, 2020.

21. Levi, M., et al., Coagulation abnormalities and thrombosis in patients with COVID-19. Lancet Haematol, 2020. 7(6): p. e438-e440.

22. Conti, C.B., et al., Bleeding in COVID-19 severe pneumonia: The other side of abnormal coagulation pattern? Eur J Intern Med, 2020.

23. Wilkerson, R.G., et al., Silent hypoxia: A harbinger of clinical deterioration in patients with COVID-19. Am J Emerg Med, 2020.

24. Cepeda-Valdes, R., et al., Cutaneous manifestations in COVID-19: Family cluster of Urticarial Rash. Clin Exp Dermatol, 2020.

25. Hunt, M. and C. Koziatek, A Case of COVID-19 Pneumonia in a Young Male with Full Body Rash as a Presenting Symptom. Clin Pract Cases Emerg Med, 2020. 4(2): p. 219-221.

26. Joob, B. and V. Wiwanitkit, COVID-19 can present with a rash and be mistaken for dengue. J Am Acad Dermatol, 2020. 82(5): p. e177.

27. Puelles, V.G., et al., Multiorgan and Renal Tropism of SARS-CoV-2. N Engl J Med, 2020. 383(6): p. 590-592.

28. Braun, F., et al., SARS-CoV-2 renal tropism associates with acute kidney injury. Lancet, 2020. 396(10251): p. 597-598.

29. Vijayan, A. and B.D. Humphreys, SARS-CoV-2 in the kidney: bystander or culprit? Nat Rev Nephrol, 2020. 16(12): p. 703-704.

30. Zahid, U., et al., Acute Kidney Injury in COVID-19 Patients: An Inner City Hospital Experience and Policy Implications. Am J Nephrol, 2020. 51(10): p. 786-796.

31. Gabarre, P., et al., Acute kidney injury in critically ill patients with COVID-19. Intensive Care Med, 2020. 46(7): p. 1339-1348.

32. Uppala, J.K., A.R. Gani, and K.V.A. Ramaiah, Chemical chaperone, TUDCA unlike PBA, mitigates protein aggregation efficiently and resists $E R$ and non-ER stress induced HepG2 cell death. Sci Rep, 2017. 7(1): p. 3831.

33. Vang, S., et al., The Unexpected Uses of Urso- and Tauroursodeoxycholic Acid in the Treatment of Non-liver Diseases. Glob Adv Health Med, 2014. 3(3): p. 58-69.

34. Khouzam, H.R., A review of trazodone use in psychiatric and medical conditions. Postgrad Med, 2017. 129(1): p. 140-148.

35. Khan, S., S.K. Komarya, and G. Jena, Phenylbutyrate and beta-cell function: contribution of histone deacetylases and ER stress inhibition. Epigenomics, 2017. 9(5): p. 711-720.

36. Quinlan, A., Assessing Cell Health: Autophagy. Bioradiations :A resourse for life science research, 2016.

37. Channappanavar, R., J. Zhao, and S. Perlman, T cell-mediated immune response to respiratory coronaviruses. Immunol Res, 2014. 59(1-3): p. 118-28.

38. Shah, V.K., et al., Overview of Immune Response During SARS-CoV-2 Infection: Lessons From the Past. Front Immunol, 2020. 11: p. 1949. 
39. Blanco-Melo, D., et al., Imbalanced Host Response to SARS-CoV-2 Drives Development of COVID-19. Cell, 2020. 181(5): p. 1036-1045 e9.

40. Cottam, E.M., et al., Coronavirus nsp6 proteins generate autophagosomes from the endoplasmic reticulum via an omegasome intermediate. Autophagy, 2011. 7(11): p. 133547.

41. Prentice, E., et al., Identification and characterization of severe acute respiratory syndrome coronavirus replicase proteins. J Virol, 2004. 78(18): p. 9977-86.

42. Rota, P.A., et al., Characterization of a novel coronavirus associated with severe acute respiratory syndrome. Science, 2003. 300(5624): p. 1394-9.

43. Ksiazek, T.G., et al., A novel coronavirus associated with severe acute respiratory syndrome. N Engl J Med, 2003. 348(20): p. 1953-66.

44. $\mathrm{Li}, \mathrm{X}$., et al., Targeting autophagy in osteoporosis: From pathophysiology to potential therapy. Ageing Res Rev, 2020. 62: p. 101098.

45. Poplawski, T., et al., Interplay between Redox Signaling, Oxidative Stress, and Unfolded Protein Response (UPR) in Pathogenesis of Human Diseases. Oxid Med Cell Longev, 2019. 2019: p. 6949347.

46. Ren, J., J.R. Sowers, and Y. Zhang, Metabolic Stress, Autophagy, and Cardiovascular Aging: from Pathophysiology to Therapeutics. Trends Endocrinol Metab, 2018. 29(10): p. 699711.

47. Uchiyama, Y., et al., Autophagy-physiology and pathophysiology. Histochem Cell Biol, 2008. 129(4): p. 407-20.

48. Fung, T.S., M. Huang, and D.X. Liu, Coronavirus-induced ER stress response and its involvement in regulation of coronavirus-host interactions. Virus Res, 2014. 194: p. 11023.

49. Fung, T.S., Y. Liao, and D.X. Liu, The endoplasmic reticulum stress sensor IRE1alpha protects cells from apoptosis induced by the coronavirus infectious bronchitis virus. J Virol, 2014. 88(21): p. 12752-64.

50. Fung, T.S. and D.X. Liu, Coronavirus infection, ER stress, apoptosis and innate immunity. Front Microbiol, 2014. 5: p. 296.

51. Bechill, J., et al., Coronavirus infection modulates the unfolded protein response and mediates sustained translational repression. J Virol, 2008. 82(9): p. 4492-501.

52. Fung, T.S. and D.X. Liu, Human Coronavirus: Host-Pathogen Interaction. Annu Rev Microbiol, 2019. 73: p. 529-557.

53. Ma, Y., et al., The Coronavirus Transmissible Gastroenteritis Virus Evades the Type I Interferon Response through IRE1alpha-Mediated Manipulation of the MicroRNA miR30a-5p/SOCS1/3 Axis. J Virol, 2018. 92(22).

54. Lim Y, et al., Human coronaviruses: a review of virus-host interactions. Diseases 2016. 4(4): p. 26.

55. Shi, C.S., et al., SARS-Coronavirus Open Reading Frame-8b triggers intracellular stress pathways and activates NLRP3 inflammasomes. Cell Death Discov, 2019. 5: p. 101.

56. Yan, M., et al., Endoplasmic reticulum stress in ischemic and nephrotoxic acute kidney injury. Ann Med, 2018. 50(5): p. 381-390.

57. Koseler, A., et al., Endoplasmic Reticulum Stress Markers in SARS-COV-2 Infection and Pneumonia: Case-Control Study. In Vivo, 2020. 34(3 Suppl): p. 1645-1650. 
58. John Bechill, et al., Coronavirus Infection Modulates the Unfolded Protein Response and Mediates Sustained Translational Repression

Journal of virology, 2008. 82(9): p. 4492-4501.

59. Krähling V, et al., Severe acute respiratory syndrome coronavirus triggers apoptosis via protein kinase $R$ but is resistant to its antiviral activity

J Virol

2009. 83(5): p. 2298-309.

60. Minakshi, R., et al., The SARS Coronavirus $3 a$ protein causes endoplasmic reticulum stress and induces ligand-independent downregulation of the type 1 interferon receptor. PLoS One, 2009. 4(12): p. e8342.

61. Ron, D. and P. Walter, Signal integration in the endoplasmic reticulum unfolded protein response. Nat Rev Mol Cell Biol, 2007. 8(7): p. 519-29.

62. Song, X., et al., Crosstalk Between Apoptosis and Autophagy Is Regulated by the Arginylated BiP/Beclin-1/p62 Complex. Mol Cancer Res, 2018. 16(7): p. 1077-1091.

63. Zhang, L. and A. Wang, Virus-induced ER stress and the unfolded protein response. Front Plant Sci, 2012. 3: p. 293.

64. Parzych, K.R. and D.J. Klionsky, An overview of autophagy: morphology, mechanism, and regulation. Antioxid Redox Signal, 2014. 20(3): p. 460-73.

65. Zheng, W., et al., ATG5 and ATG7 induced autophagy interplays with UPR via PERK signaling. Cell Commun Signal, 2019. 17(1): p. 42.

66. Høyer-Hansen $M$ and J. M., Connecting endoplasmic reticulum stress to autophagy by unfolded protein response and calcium. Cell Death Differ., 2007. 14(9): p. 1576-82.

67. Ozcan, U., et al., Chemical chaperones reduce ER stress and restore glucose homeostasis in a mouse model of type 2 diabetes. Science, 2006. 313(5790): p. 1137-40.

68. Choi, S.E., et al., A chemical chaperone 4-PBA ameliorates palmitate-induced inhibition of glucose-stimulated insulin secretion (GSIS). Arch Biochem Biophys, 2008. 475(2): p. 10914.

69. Doerflinger, M., et al., Chemical chaperone TUDCA prevents apoptosis and improves survival during polymicrobial sepsis in mice. Sci Rep, 2016. 6: p. 34702.

70. Lee, Y.Y., et al., Tauroursodeoxycholate (TUDCA), chemical chaperone, enhances function of islets by reducing ER stress. Biochem Biophys Res Commun, 2010. 397(4): p. 735-9.

71. Wang, D., et al., The Chemical Chaperone, PBA, Reduces ER Stress and Autophagy and Increases Collagen IV alpha5 Expression in Cultured Fibroblasts From Men With X-Linked Alport Syndrome and Missense Mutations. Kidney Int Rep, 2017. 2(4): p. 739-748.

72. Grein, J., et al., Compassionate Use of Remdesivir for Patients with Severe Covid-19. N Engl J Med, 2020. 382(24): p. 2327-2336.

73. Liu, S.T.H., et al., Convalescent plasma treatment of severe COVID-19: a propensity scorematched control study. Nat Med, 2020. 26(11): p. 1708-1713.

74. Das, I., et al., Glucocorticoids alleviate intestinal ER stress by enhancing protein folding and degradation of misfolded proteins. J Exp Med, 2013. 210(6): p. 1201-16.

75. Oray, M., et al., Long-term side effects of glucocorticoids. Expert Opin Drug Saf, 2016. 15(4): p. 457-65. 
bioRxiv preprint doi: https://doi.org/10.1101/2021.02.04.429769; this version posted February 4, 2021. The copyright holder for this preprint (which was not certified by peer review) is the author/funder. All rights reserved. No reuse allowed without permission. 


\section{Figure Legends}

Figure 1. Upregulation of BiP/GRP78 in HEK293T cells over-expressing the SARSCoV-2 Spike protein. HEK293T cells were transfected with pcDNA3.1-SARS2-Spike and the protein levels of (a) BiP/GRP78 and (b) phosphorylated elF2 $\alpha$ (pelF2 $\alpha$ ) were determined by Western blotting using specific antibodies 24 hours after transfection. Quantified values of the specific protein abundance, which were normalized to the abundance of GAPDH, were included at the right panel.

Figure 2. SARS-CoV-2 Spike protein over-expression triggers autophagy through UPR. (a) HEK293T cells were transfected with pcDNA3.1-SARS2-Spike and the protein levels LC3I and LC3-II were determined by Western blotting using anti-LC3B antibody after 24 hours. Values were normalized to GAPDH. The ratio of LC3-II over GAPDH is graphically represented on right. (b) Cell viability of the transfected cells was evaluated by MTT assay and the quantified values were normalized to non-transfected controls.

Figure 3. UPR modulator treatment reduced levels of ER stress, UPR and autophagy. HEK293T cells were transfected with pcDNA3.1-SARS2-Spike and 4 hours after transfection, the cells were treated with the selected UPR modulators, Salubrinal $(50 \mu \mathrm{M}), 4-P B A(2 \mathrm{mM})$, TUDCA $(100 \mu \mathrm{M})$, DBM $(50 \mu \mathrm{M})$ and Trazadone $(50 \mu \mathrm{M})$ for 24 hours. The protein levels of (a) BiP/GRP78, (b) phosphorylated elF2a were determined by Western blotting using specific antibodies. Values were normalized to that abundance of GAPDH and were presented in the graphs on the right. (c) The protein levels LC3-II with UPR modulator treatment were determined by Western blotting and normalized to the abundance of GAPDH. The ratio of LC3 II over GAPDH is graphically represented on right. * represents significant values compared to control. ** represents significant values compared to HEK293T + SARS-CoV-2 Spike. 
Fig. 1
a)
BiP/GRP78

GAPDH

b)

pelF2 $\alpha$

GAPDH
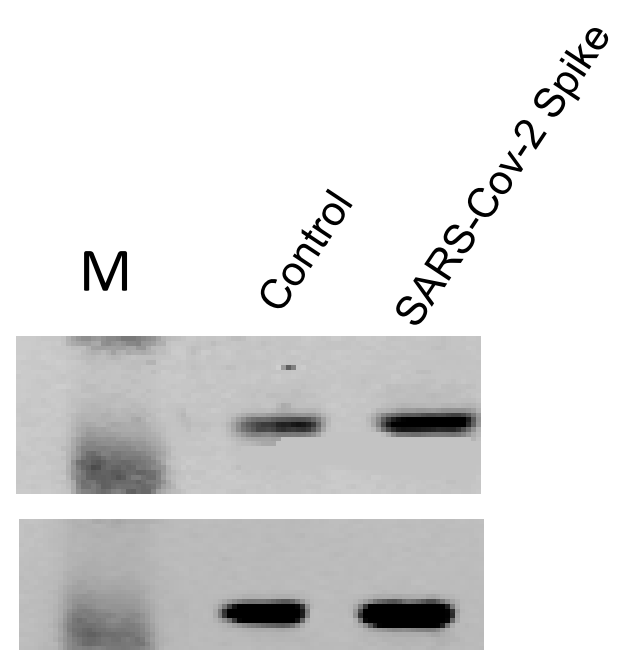
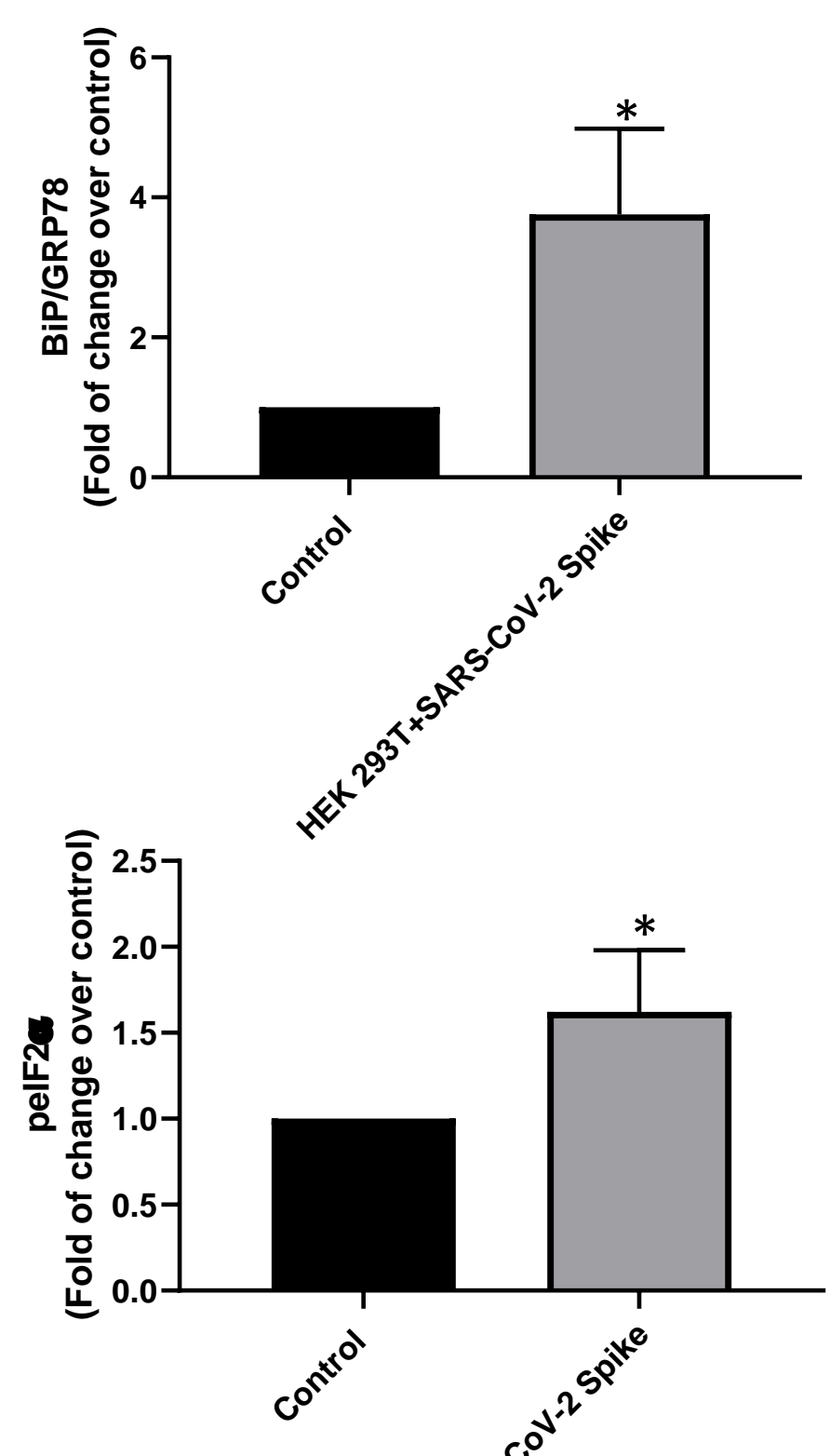
Fig. 2

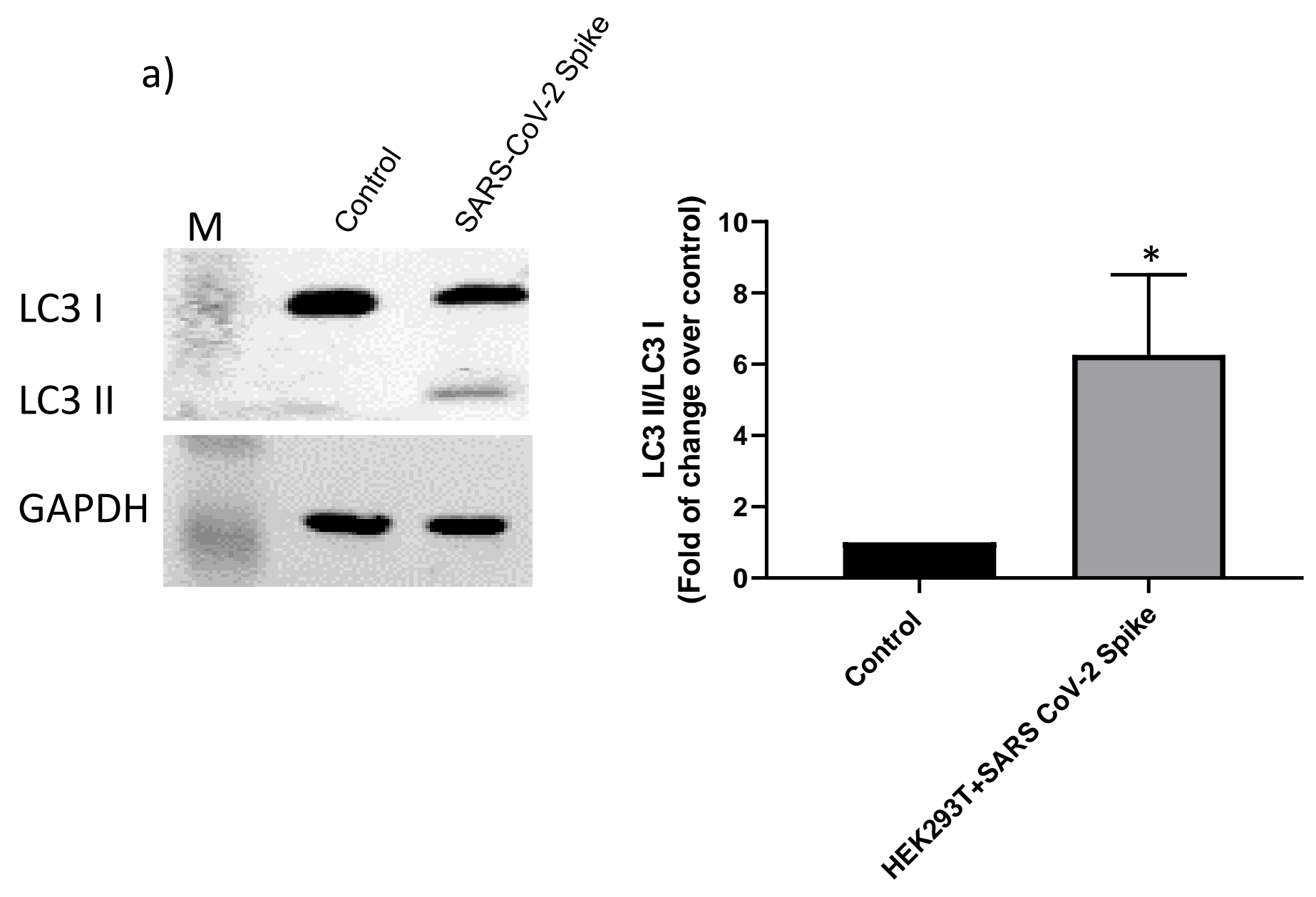

b)

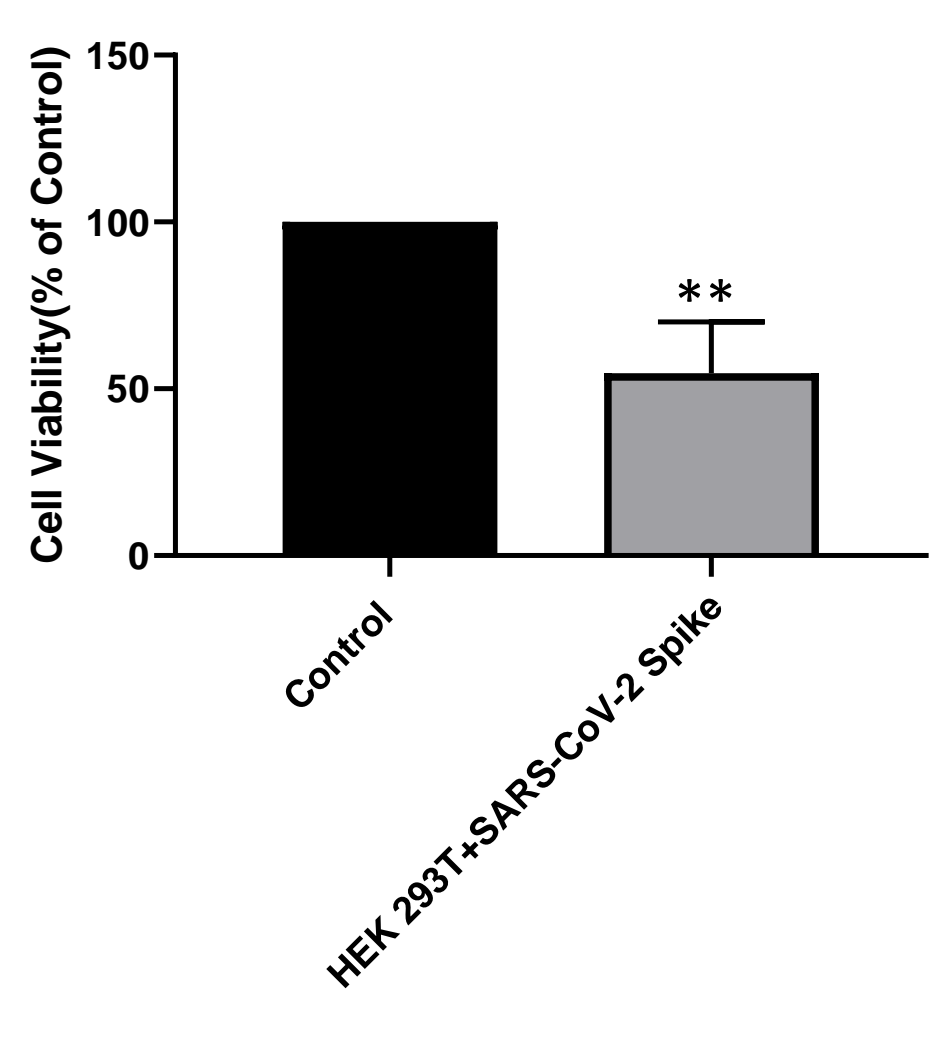




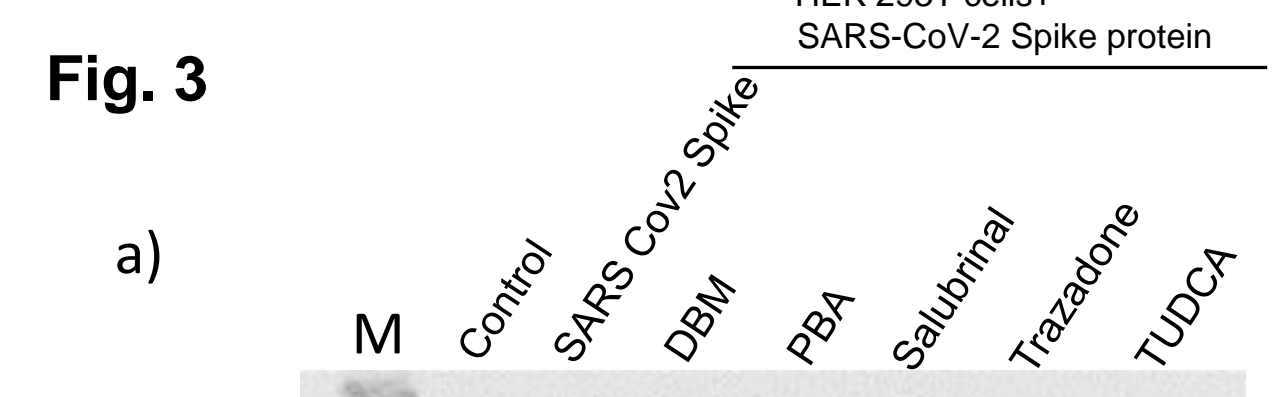

BiP/GRP78

GAPDH b)
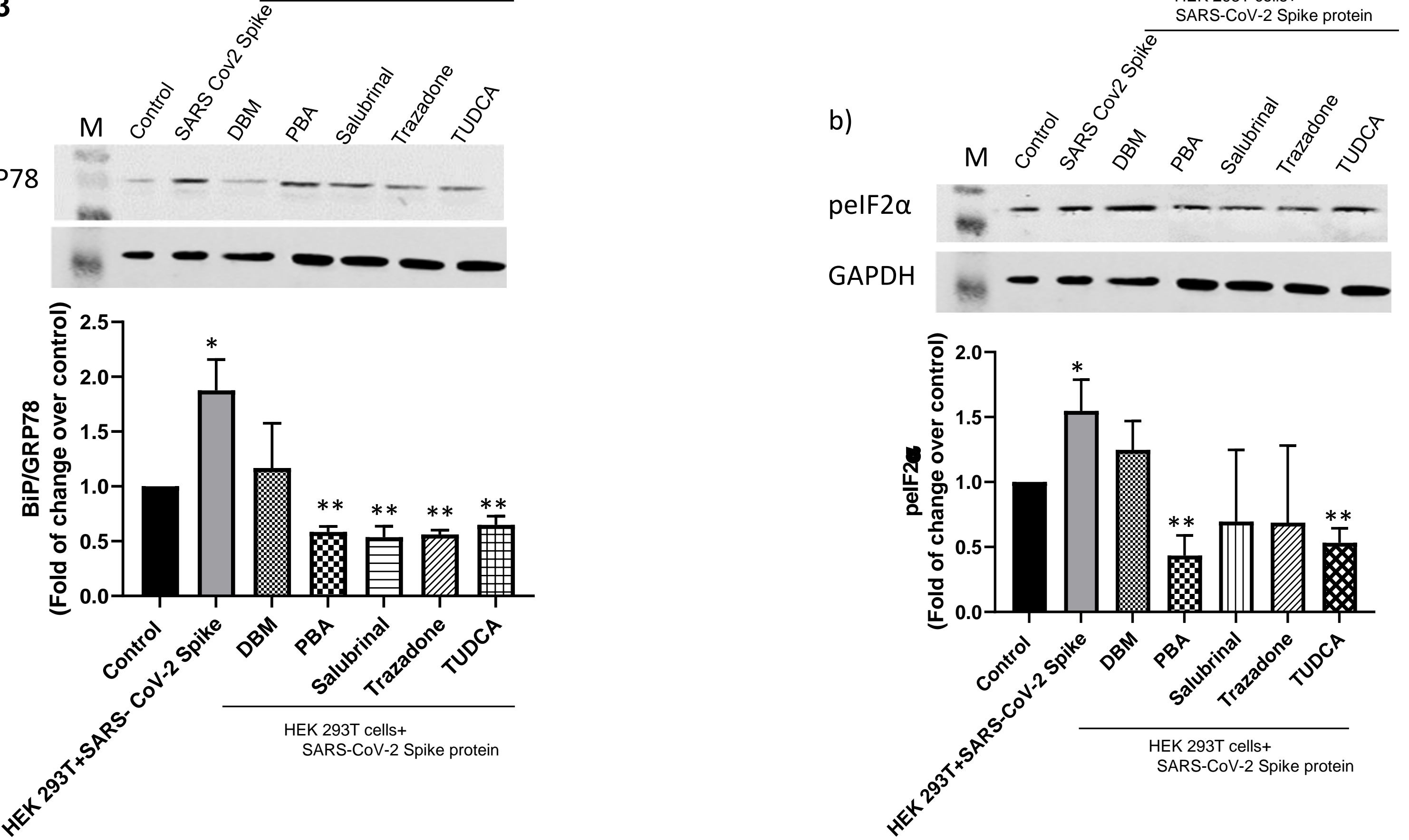
Fig. 3

c)

HEK 293T cells+

SARS-CoV-2 Spike protein
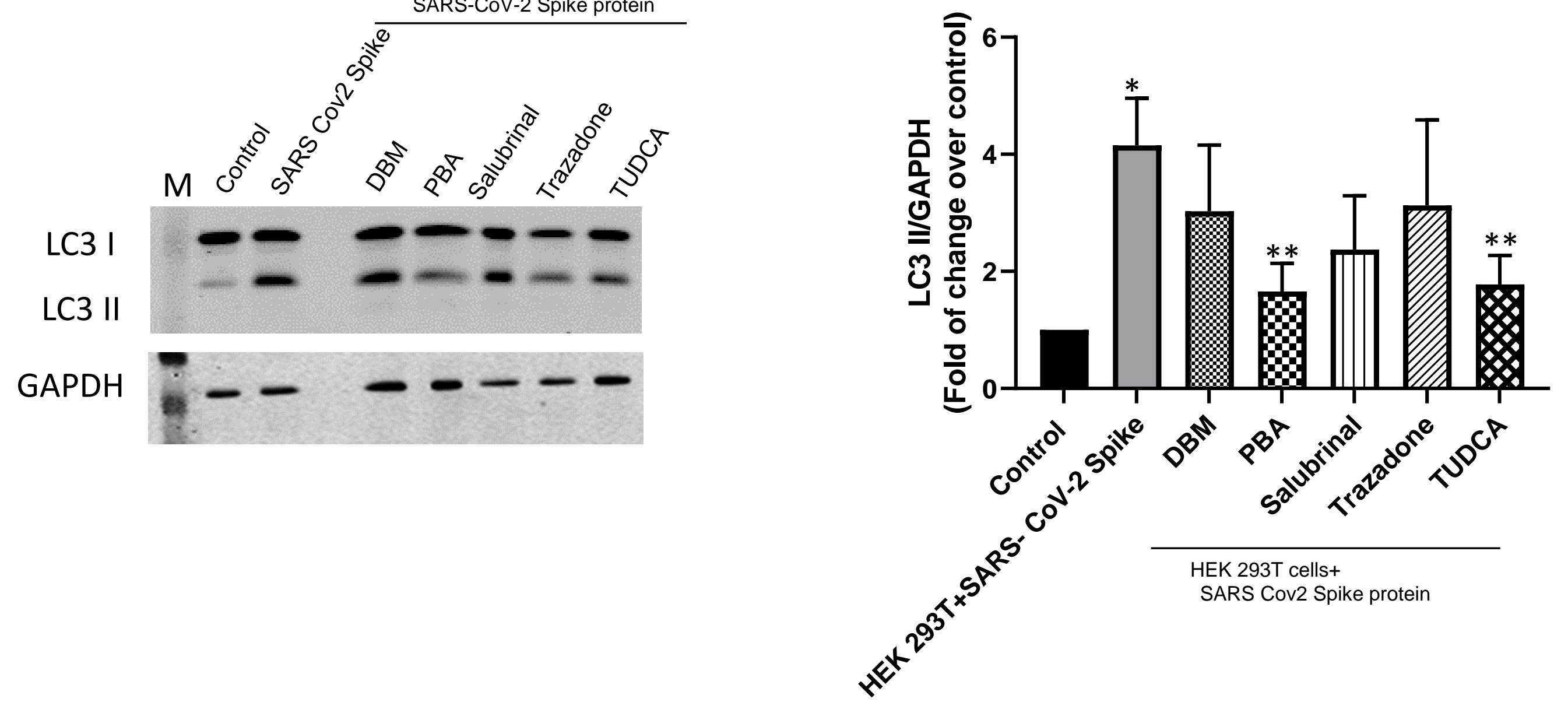